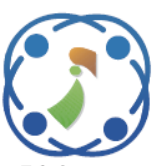

\title{
A Formal Analytical Modeling and Simulation of Wireless Sensor Home Network
}

\author{
Jwaone Gaboitaolelwe ${ }^{1} \quad$ Adamu Murtala Zungeru ${ }^{1 *}$ \\ Nonofo Ditshego ${ }^{1} \quad$ Thabo Semong ${ }^{2}$ \\ Joseph Chuma ${ }^{1}$ \\ ${ }^{1}$ Department of Electrical, Computer and Telecommunication Engineering, \\ Botswana International University of Science and Technology, Private Bag 16, Palapye, Botswana \\ ${ }^{2}$ Department of Computer Science and Information Systems, \\ Botswana International University of Science and Technology, Private Bag 16, Palapye, Botswana \\ * Corresponding author's Email: zungerum@ biust.ac.bw
}

\begin{abstract}
Technological advances for low-cost Wi-Fi applications such as 'the wireless sensor network (WSN) deployment' are at an advanced stage of acceptance, especially in smart home environments. The application of this kind of network is referred to as a wireless sensor home network (WSHN). In literature, the evaluation of customers' home wireless network performance level is usually done via real-time experiment or by experience to test the network's performance, leading to network vendors able to gain reference performance for: improvement of their network capacity and the adoption of the emerging requirement of Wi-Fi services. However, the usual practice of comparison of energy consumption in WSHN applications is by developing WSHN systems for information transmission and reception in the home network to measure delay, throughput and energy consumption performance metrics using heuristics and intuition approaches. This work shows that real-time-based performance measurements of WSHN systems should be first carried out using the formal analytical method; before simulation and real-time experiments. Use of analytical method in WSHN provides users with a more defined idea of the kind of results to expect from their network simulations. To this end, this work presents an analytical method based on energy consumption for WSHN grid-based and line-based topology for the same metrics, with a description of each of the networks, which is first of its kind in WSHN. The work is further strengthened through simulation results. The results of our mathematical analysis show a variation of network energy consumption for single-hop and multi-hop communication for reference purposes for future WSHNs. The mathematical analysis results are futher validated by the simulation results of WSNs comprising of nodes using ESP32 microcontrolers RF specifications; where it shows that in most cases, using single-hop communication might cause excessive energy consumption in the network. This being more pronounced when the distance apart of the smart devices is above 50feet or the network nodes is above 20; more also, when the packet size is above 50 bits. In this case, multi-hop communication it is seen to be of preferable communication at a higher number of nodes, larger packet size and longer distance between devices. However, if the network size is small, there is little difference in the energy consumption between the two methods and it is in such networks where single-hop is preferred due to the reduced complexity of the network structure and small delay time of which promotes responsiveness of the network.
\end{abstract}

Keywords: Smart home, Wireless sensor networks, Wireless sensor home networks, Grid network, Linear network.

\section{Introduction}

Advances in wireless sensor home network (WSHN) systems have drawn researcher's attention to dependable and embedded smart devices in-home network applications. This is partly due to advancements in wireless communication technologies and Micro-Electro-Mechanical Systems (MEMS) technology that have enabled the development of smaller and more energy-efficient smart sensors. However, despite these advances, numerous challenges are still faced by WSHN. Such challenges include converting normal devices into smart devices that can integrate with the WSHN, the energy consumption of the smart devices, limited 
storage capacity and limited distance of transmission of the communication modules like the Wi-Fi modules. Despite these challenges, the demand for smart devices in smart homes and smart buildings continues to grow as our lives become more entangled with technology.

In the typical establishment of smart homes and buildings; wireless sensor network technology used in WSHN provides a good framework that can be used to easily and inexpensively incorporate smart device technology into existing buildings without the need for dedicated communication and power cables. However, the lack of dedicated wired infrastructure for sensors and device communication in wireless networks (especially low power networks) face problems of reliability and transmission range. Unlike wired networks, wireless networks particularly those in urban areas experience unpredictable wireless environments of which affect their reliability. Hence, to assess the performance of the proposed system, modeling and simulation before prototyping of the WSHN are required.

The next subsections of the paper will present the analytical (Mathematical) models and simulation environment parameters considered in the design of the proposed system. The method of analysis is inspired by the works of [1], and as used in [2,3], and hence will proceed similarly. The work proceeds as follows: 1. Mathematical analysis of node distribution and connectivity; 2. Network reliability dependent on network topology and node energy consumption; 3 . Network simulation.

There are two main contributions of the paper. First being an analytical method for WSHN gridbased topology based on energy consumption and line-based topologies for the same metrics of which is the first of its kind. Second being the strengthening of the work through simulation of the networks.

The rest of the paper organized as follows: Section 2 discusses work related to this research. Section 3 describes the wireless home network and some of the definitions including node distribution and connectivity, energy modeling and network reliability. Section 4 shows and explains the network simulation and the results obtained. Based on the analysis and simulation results, a short discussion in the implementation of a WSHN is shown in Section 5 and the paper is concluded in Section 6, with highlights of few remarks.

\subsection{Related work}

Some researchers [4-6] propose analytical modeling of WSN lifetime particularly focused on the remaining energy of nodes as a single unit and as a system. In their work, other factors such as sensors' positions in relation to other sensors, the link quality, connectivity and coverage of the sensor network are also taken into consideration in the creation of the models. The purpose of the models being used is to acquire evaluation metrics for efficiency, effectiveness, and performance of the designed protocols and algorithms for WSN.

The authors in $[7,8]$ researched the analysis of reliability in WSN. Both papers model WSN using connected graphs but they differ in that [7] focuses on a general understanding of how different network topology characteristics such as connectivity, average degree, diameter, average path length and clustering coefficient affect network reliability. Whereas [8] strives for a more quantitative evaluation of the network reliability with the idea of quantifying the importance of nodes in a network since depending on the topology, certain nodes have a march larger impact or contribution in network reliability than other nodes.

WSN models are also used in the works of [911] to make a comparison between single-hop and multi-hop architectures and routing protocols and how they impact network throughput, delay, reliability and lifetime. The works of [9] place more emphasis on analyzing the design constraints of each routing method and based on their work, further recommend the applications suitable for each method. As for [10, 11], importance is placed in investigating the differences in the performance of the two methods.

Some research work on energy-aware and battery aware WSNs can be found in [12-15] respectively. The approach used in [12] is that of finding a path from a source node to a destination node that minimizes the total consumed energy in a network by formulating a constrained programming problem that uses both the locations of the sensor nodes and data transmission pattern. In [13], the proposed analytical model takes into consideration the characteristics of batteries used in nodes and uses them to find optimal node sleep intervals with a trade-off between the energy dissipation used in idle listening and sending of a preamble. To support the works of energy/battery aware WSNs such as that of $[12,13]$, authors [14, 15] propose software-based approach to estimate the state of charge, lifetime and voltage of batteries in WSN nodes based on the use of a temperature-dependent analytical battery models. In other works, such as that of [16-18], 


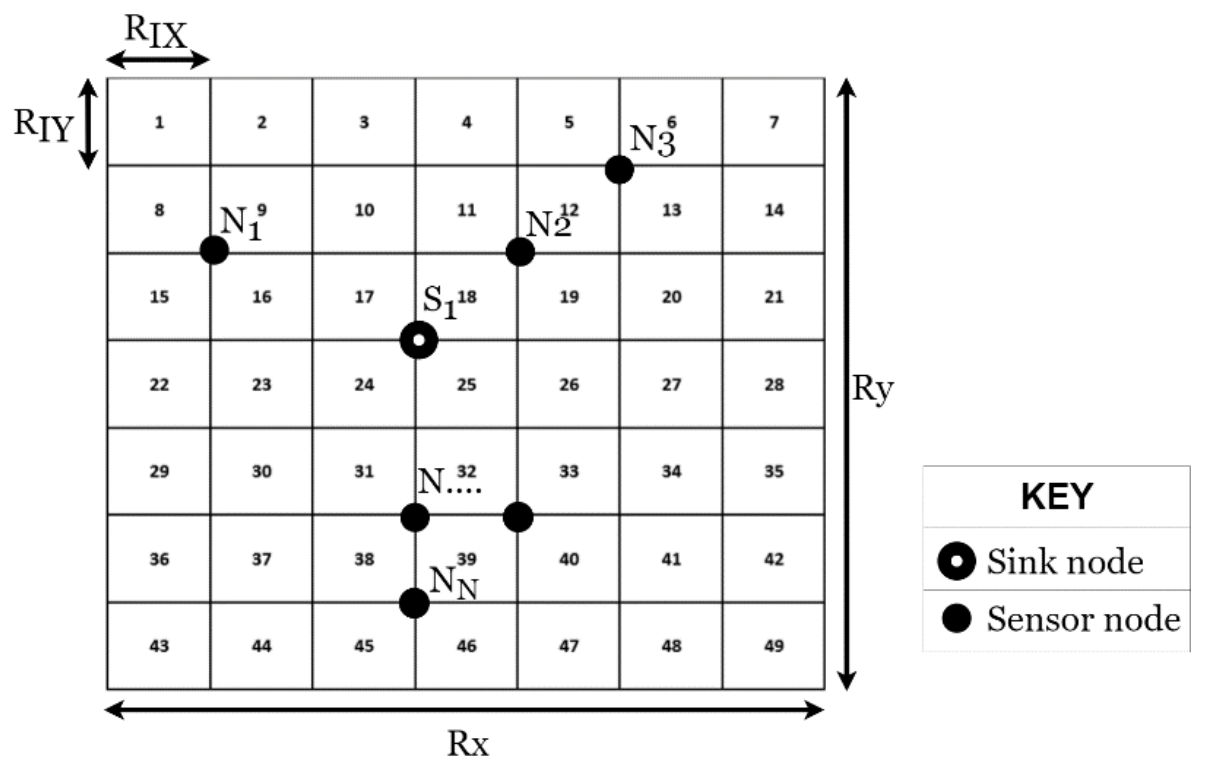

Figure. 1 Structure of a wireless sensor home network with a grid of x-y network topology

instead of using the traditional battery-powered sensor networks, which exhibit limited lifetime. The authors propose the analysis and modeling of solar energy harvesting WSNs (EH-WSN) for use in the operation of self-powered and self-sustained WSNs.

Unlike most papers that use minimum hop count as a performance metric in route path selection, authors in [19] consider the use of rate aware routing protocols to minimize network layer transmission time as a performance matrix. The papers intend to maximize the network lifetime and to minimize the total energy cost of a WSN. Other works such as that of [20], the author extend their previous work of grid-based hybrid network deployment approach, in which merge and split technique has been proposed to construct network topology. In their new work, they use analytical network process method for Cluster Head selection in a WSN for selection with better understanding of the dependencies of different components involved in the evaluation process.

This work shows that real-time-based performance measurements of WSHN systems should be first carried out using the formal analytical method; before simulation and real-time experiments. Use of analytical method in WSHN provides users with a more defined idea of the kind of results to expect from their network simulations. Similar to the works before it such as those mentioned above, this work uses analytical modeling and simulation, but contrary to other works, this work presents an analytical method based on energy consumption for WSHN grid-based and line-based topology for the same metrics, with a description of each of the networks, which is first of its kind in WSHN. The work is further strengthened through simulation results.

\section{Sensor home network description and definitions}

\subsection{WSHN description: Node distribution and connectivity}

Assuming a network comprising of one (1) sink node, $S_{1} . N$ number of sensors nodes ranging from $N_{1}, N_{2}, N_{3}, \ldots$ up to node $N_{N}$. The nodes have static locations and are uniformly randomly distributed over a grid model region of the area, $A$, comprising of perimeter dimensions $\mathrm{R}_{\mathrm{X}}$ and $\mathrm{R}_{\mathrm{Y}}$ and inter grid spacing of $R_{I X}, R_{I Y}$ along with the $X$ and $Y$ directions respectively. In such a network, the expected number of nodes that can be found in a region of the network is described by the node density, $P$, expressed as $P=\frac{N_{N}}{A}$. Fig. 1 is an illustration of the described grid network.

For connectivity amongst the nodes of the wireless sensor home network, this work adopted a model similar to the work in [1-3] as a simple radio link, which is used for the analysis of the network. The nodes in the network carrying a radio link model have a finite transmission range, $d_{t x}$ and are fitted with an Omni-directional antenna, allowing for equal wireless transmission in all directions. Restriction of the node wireless transmission range is added to signify the bandwidth limitation experienced in sensor networks.

All nodes in the network are capable of bidirectional communication; therefore they can 


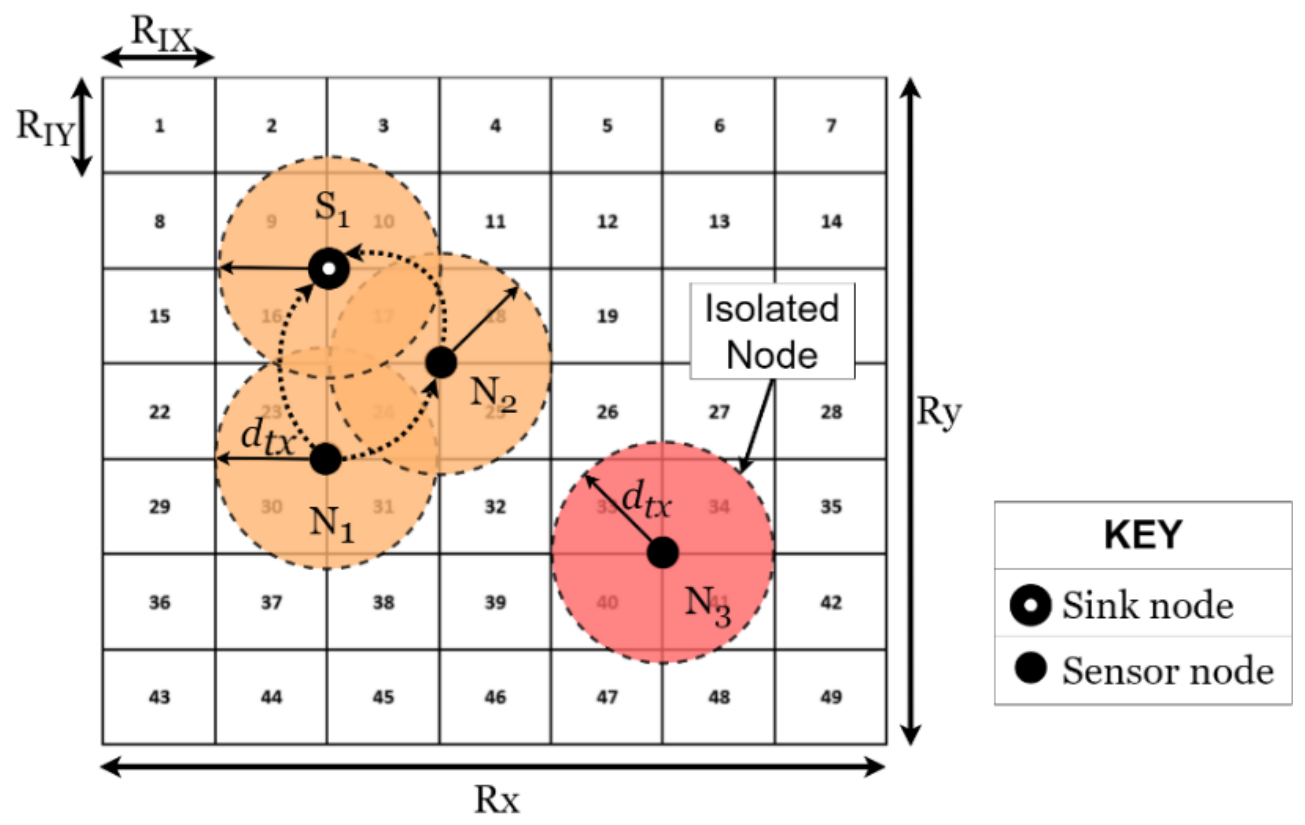

Figure. 2 Wireless sensor network model with an isolated node

both transmit and receive n-bit data packets. Any node in the grid network can act as a source node so long as it has information to be transmitted to the sink node. For communication to occur between nodes, only nodes that are within the transmission range of each other can establish a successful wireless link and communicate. Nodes that are completely isolated from others cannot form a wireless link and therefore are unable to communicate with other nodes. This can be seen in Fig. 2, where sink node $S_{1}$ is within the transmission range of nodes $N_{1}$ and $N_{2}$, hence communication between the nodes is possible. However, node $N_{3}$ is beyond the transmission range of any node. This makes it isolated and unable to form a wireless link for communication.

Wireless transmission between nodes occurs via wireless channels. The source node transmits a signal at power, $P_{t x}$ at zero distance, $d=0$ from a source node. The transmitted signal power undergoes attenuation in wireless channel medium and is received at the destination node as power, $P_{r x}$, at distance, $d$, from the source node. The received power, $P_{r x}$ is inversely proportional to attenuation or path loss, $d^{\prime} P_{t x}$ of the transmitted signal. Gamma, $\gamma$ represents the path loss exponent and varies between 2 and 4 depending on the environment [2-4]. For successful transmission and reception, it is assumed that the transmitted signal, $\mathrm{dP}_{\mathrm{tx}}$ is greater than the receiver sensitivity $\left(P_{r x}\right)$. Therefore, $P_{t x}\left(d=d_{t x}\right) \geq$ $P_{r x}$, for successful reception of a signal.

Data transmitted by a source node can reach a sink node in two ways. Either direct transmission to the sink node in a single hop or it can be forwarded through other sensor nodes in a series of hops until it reaches the sink node. This is also illustrated in Fig. 2 , seeing how data packets from node $N_{l}$ are transmitted directly to the sink node by single-hop or reach the sink node by taking the path via node $\mathrm{N}_{2}$ of which leads to 2(two) hops.

For both transmission and reception of data packets (event data) from or to other nodes in a network, energy is required by a grid node to carry out the task. The energy used up by nodes in a single hop event forwarding operation can be expressed as:

$$
E_{T}=E_{t x}+E_{r x}
$$

Where $E_{T}$ is the total expended energy, $E_{t x}$ is the energy used in data transmission, and $E_{r x}$ is the energy used when receiving data [5].

When nodes of the same communication range transit data between each other, the amount of transmission energy required is proportional to the size of the data and the square of the distance between the nodes [1-3]. Since energy is expended by nodes when both when transmitting and receiving data packets to and from other nodes in a network (whether directly to the target node or indirectly through a series of hops). An arrangement is required that can consider and link energy or power consumption when forwarding data to the distance between the source and sink node. This way, one can compare single hop and multiple hop transmission.

Consider the grid network example in Fig. 3, where source node $N_{l}$ is sending data to sink node $S_{l}$. 


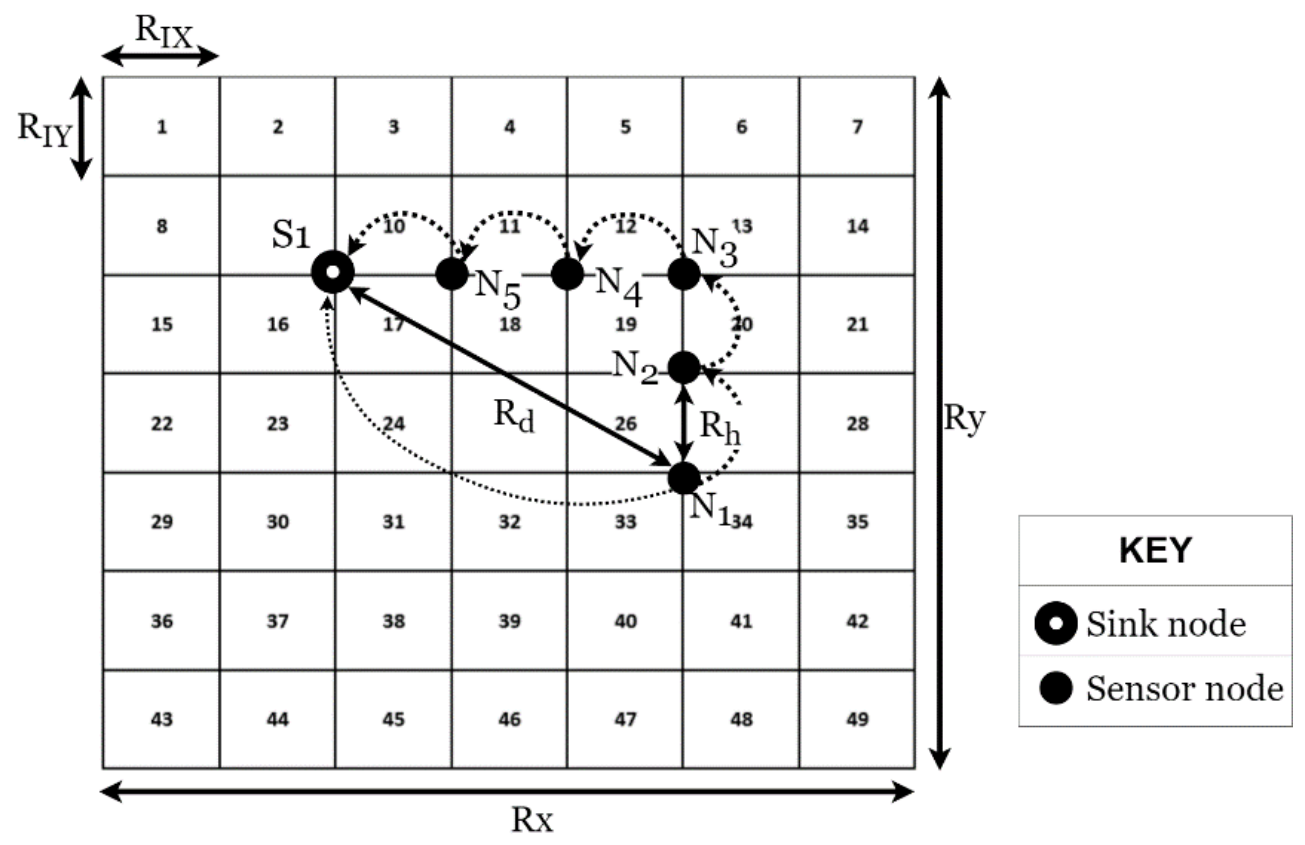

Figure. 3 Single hop and multi-hop routing in $\mathrm{x}-\mathrm{y}$ grid topology wireless sensor network

If the distance between the sink node and source node is short and multiple hops are used to transmit data, just like data is transmitted from $N_{1}$ to $S_{1}$ through $N_{2}$ to $N_{5}$. The number of forwarding hops to the sink node will increase energy consumption since multiple transmissions and receptions are performed. Then again, if the distance between the source and sink nodes is too high and a data packet is transmitted directly to the sink node, more energy will be used by the source node to send the signal across the large space.

In determining the energy costs of using either method of data forwarding, whether direct or indirect. Information such as distances and the number of hops between nodes is required to perform the calculations. Again, using Fig. 3 as an example, the distance between consecutive grid nodes, $R_{h}$, such as between nodes $N_{l}$ and $N_{2}$ can be shown as:

$$
R_{h}=\left(\left(R_{I X 1}-R_{I X 2}\right)^{2}+\left(R_{I Y 1}-R_{I X 2}\right)^{2}\right)^{\frac{1}{2}}
$$

Where $R_{I Y}$ and $R_{I X}$ are the grid distance coordinates for the nodes in the $\mathrm{y}$ and $\mathrm{x}$ directions respectively. The total number of vertical and horizontal hops taken to reach the sink node from the source node will then be $Y_{h}$ and $X_{h}$ respectively. Leading to:

$$
\begin{aligned}
& X_{h}=\frac{\left(R_{I X \min }-R_{I X \max }\right)^{2}}{R_{h}} \\
& Y_{h}=\frac{\left(R_{I Y \min }-R_{I y \max }\right)^{2}}{R_{h}}
\end{aligned}
$$

Having the distance between consecutive nodes and the number of hops between the source and sink node. The direct transmission distance, $R_{d}$, from the source to the sink node, is taken as:

$$
R_{d}=\left(\left(R_{h} X_{h}\right)^{2}+\left(R_{h} Y_{h}\right)^{2}\right)^{\frac{1}{2}}=R_{h}\left(X_{h}{ }^{2}+Y_{h}{ }^{2}\right)^{\frac{1}{2}}
$$

To calculate the average cost of communication, $T_{\text {avg }}$, of forwarding data using multiple hops from the source to sink node. $T_{\text {avg }}$ is expressed such that it shows the average energy expenditure for every unit distance as:

$$
T_{\text {avg }}=\frac{\sum_{\left(X_{h}+Y_{h}\right) h o p s}\left(E_{t x}+E_{r x}\right)}{R_{d}}
$$

Assuming a first order radio model as illustrated in Fig. 4, per [21-23]. The data transmission energy in a node, $E_{t x}$, can be attributed to two factors. These being the energy used by the radio transmitter circuitry, taken as radio dissipation, $E_{\text {elec }}(\mathrm{J} / \mathrm{bit})$ and the transmit amplifier energy, $E_{a m p}\left(\mathrm{~J} / \mathrm{bit}^{*} \mathrm{~m}^{2}\right)$. Hence, a single-hop transmission of a data packet of size $s$ bits, over $R$ distance, using the first order radio model and taking the free space path loss exponent as, $\gamma=2$, leads to data transmission energy, $E_{t x}$, of:

$$
\begin{aligned}
& E_{t x}(s, R)=E_{\text {tx-elec }}(s)+E_{\text {tx-amp }}(s, R) \\
& E_{t x}(s, R)=s E_{\text {elec }}+s R^{\gamma} E_{\text {amp }},
\end{aligned}
$$




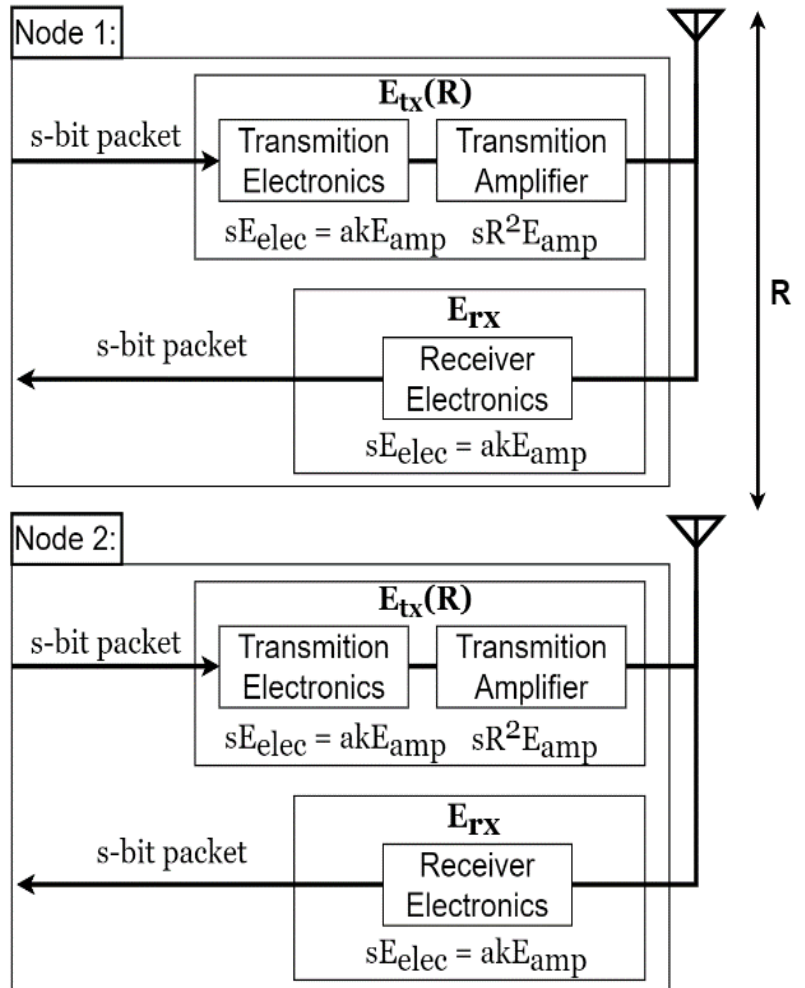

Figure. 4 Single hop transmission first order radio model

Taking alpha, $\alpha$, as an amplification factor, where $\alpha=\frac{E_{\text {elec }}}{E_{\text {amp }}}$, this gives;

$$
\begin{gathered}
E_{t x}(s, R)=s \alpha E_{a m p}+s R^{\gamma} E_{a m p} \\
E_{t x}(s, R)=s E_{a m p}\left(\alpha+R^{2}\right)
\end{gathered}
$$

On the other hand, when receiving data from a single hop transmission of data packets $s$-bits in size. Only the radio dissipation, $E_{\text {elec }}(\mathrm{J} / \mathrm{bit})$, by the radio receiver circuitry is needed. Hence leading to data reception energy, $E_{r x}$, of:

$$
E_{r x}(s)=E_{r x-e l e c}(s)=s E_{\text {elec }}=\alpha s E_{\text {amp }}
$$

By substituting and expanding both Eqs. (10) and (11) into Eq. (6), the average energy expended for event forwarding per unit distance can be expressed as:

$$
\begin{aligned}
& T_{a v g}=\frac{\left(X_{h}+Y_{h}\right)\left[\alpha s E_{a m p}+s R^{2} E_{a m p}+\alpha s E_{a m p}\right]}{R \times\left(X_{h}{ }^{2}+Y_{h}{ }^{2}\right)^{\frac{1}{2}}} \\
& T_{\text {avg }}=\frac{\left(X_{h}+Y_{h}\right) s E_{a m p}\left(2 \alpha+R^{2}\right)}{R \times\left(X_{h}{ }^{2}+Y_{h}{ }^{2}\right)^{\frac{1}{2}}}
\end{aligned}
$$

To find the upper bound for energy consumption in a static $R_{X}$ by $R_{Y}$ grid-based network, where $X_{h}, Y_{h}, n$, and Eamp are constant, the worst-case scenario of Eq. (13) is considered as:

$$
\begin{gathered}
T^{\prime}{ }_{\text {avg }}=\frac{d\left(T_{\text {avg }}\right)}{d R}=0 \\
T^{\prime}{ }_{\text {avg }}=\frac{R_{d}}{R \times R_{d}}\left[\frac{\left(X_{h}+Y_{h}\right) s E_{a m p}\left(2 \alpha+R^{2}\right)}{R \times\left(X_{h}{ }^{2}+Y_{h}{ }^{2}\right)^{\frac{1}{2}}}\right]=0
\end{gathered}
$$

That is to say;

$$
\frac{\left(X_{h}+Y_{h}\right) s E_{a m p}}{\left(X_{h}^{2}+Y_{h}^{2}\right)^{\frac{1}{2}}}\left[\frac{R_{d}}{R \times R_{d}}\left[\frac{\left(2 \alpha+R^{2}\right)}{R}\right]\right]=0
$$

or;

$$
\frac{R_{d}}{R \times R_{d}}\left[\left(2 \alpha R^{-1}+R\right)\right]=0
$$

Leading to;

$$
\begin{aligned}
& -2 \alpha R^{-2}+1=0 \\
& -2 \alpha=-R^{2}
\end{aligned}
$$

Finally;

$$
R=(2 \alpha)^{1 / 2}
$$

An output that implies that the average cost of transmission happens when $R=(2 \alpha)^{1 / 2}$.

\subsection{Wireless sensor network description: Energy modeling and network reliability}

When dealing with energy and reliability of a WSN, according to [1, 23-25], [26-32], reducing the network energy consumption can improve network reliability. This is due to that in most applications of WSN, and nodes are battery-powered. High energy consumption leads to fast depletion of the node batteries of which leads to reduced network lifetime. Thus, by analyzing the energy consumed during sending and receiving of data between nodes, network reliability can be improved by selecting the right topologies and protocols.

In a single-hop communication approach, data is sent directly from a source to a sink node. This is shown in Fig. 5, where the sensor node (1) is sending directly to the sink node. If the distance between the two (2) nodes is large, so is the amount of energy used by the source node to transmit the data to the sink. 


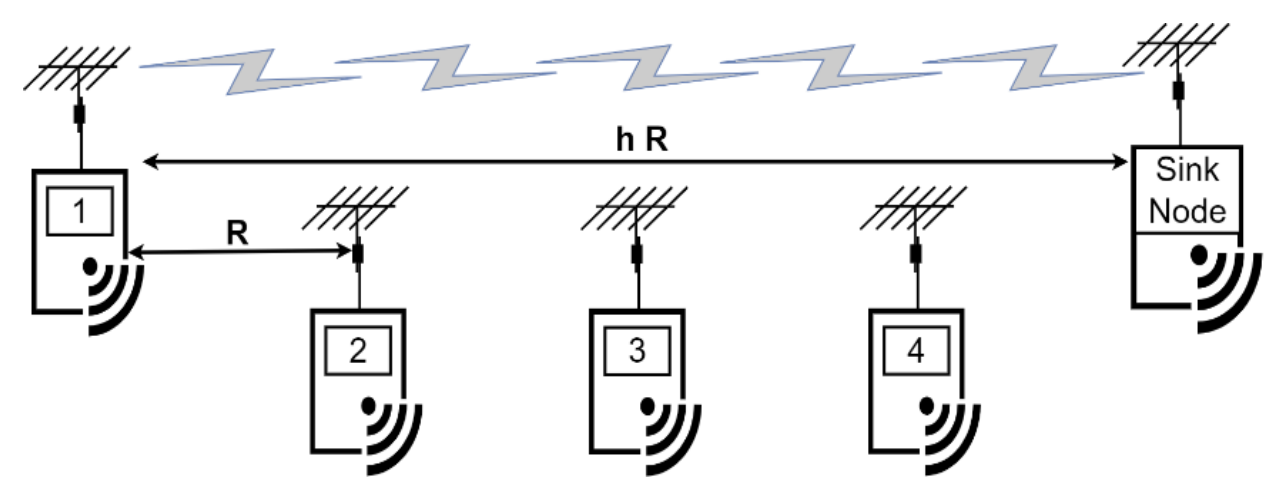

Figure. 5 A Single hop approach of Wireless Sensor Home Network

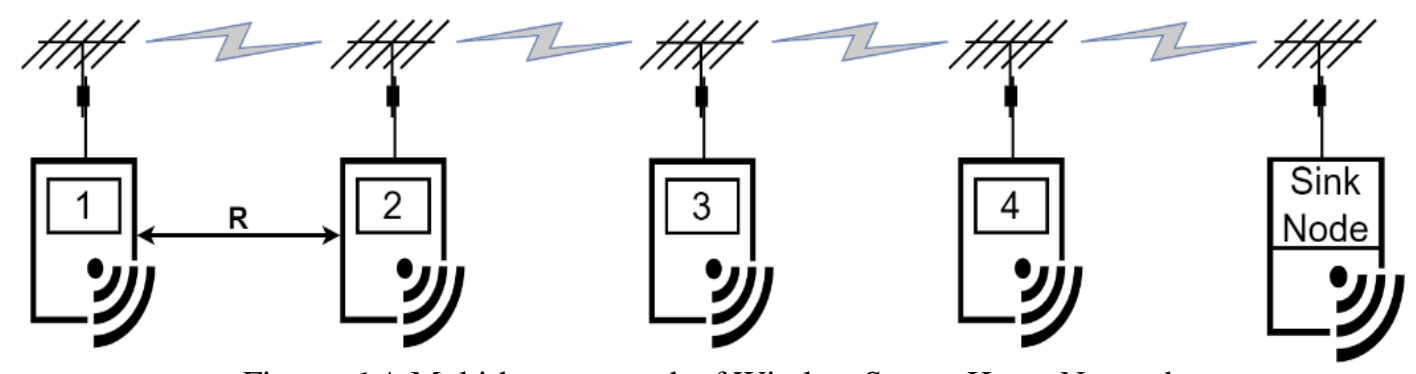

Figure. 6 A Multi-hop approach of Wireless Sensor Home Network

For a multi-hop communication approach, data from a source node reaches the sink node through a chain of hops by using other network sensor nodes as relays to forward the data until it reaches the sink node. This can be seen in Fig. 6, where data from a node (1) reaches the sink node by using node (2) to a node (4) as relays. If the distance between the source and sink node is large; the number of hops required to transmit the message also increases, hence more energy is used.

In terms of energy dissipation, the main difference between the two approaches is how the transmission cost affects the nodes in the network. With the single-hop approach, all the energy for data transmission is provided by the participating source node. Therefore, its battery will reduce at a faster rate, leading to a reduction in network lifetime. Whereas, for a multi-hop approach, the cost of transmission is distributed across the source node and the intermediate nodes. However, depending on the energy costs of the radio electronics and transmitter amplifier, the total energy expended in sending data in a multi-hop approach may be higher than a direct transmission to the sink node.

To illustrate the above statement, using Fig. 6, consider the energy used in the transmission of $s$-bit of data between a source and sink node with a distance of $(h) R$ between them, where $h$, is number of hops from source to sink node and distance between adjacent network nodes is represented by $R$. In a multi-hop routing approach, data transmitted from a source node to a sink node at a distance $(h) R$ is transmitted $h$ times and received $h-l$ times to reach its destination. Therefore, with such an approach, the energy consumption can be expressed as:

$$
\begin{aligned}
& E_{M H R}=E_{t x}+E_{r x} \\
& E_{M H R}=h E_{t x}(s, R)+(h-1) E_{r x}(s)
\end{aligned}
$$

Substituting Eq. (10) and Eq. (11) into Eq. (22), results in:

$$
\begin{aligned}
& E_{M H R}=h\left(s E_{a m p}\left(\alpha+R^{2}\right)\right)+(h-1) \alpha s E_{a m p} \\
& \quad=h s E_{a m p}\left(\alpha+R^{2}\right)+(h-1) \alpha s E_{a m p} \\
& \quad=h \alpha s E_{a m p}+h s R^{2} E_{a m p}+h \alpha s E_{a m p}-\alpha s E_{a m p} \\
& =s E_{a m p}\left(2 \alpha h+h R^{2}-\alpha\right)
\end{aligned}
$$

If a direct routing approach is used, as seen in Fig. 5. The energy consumption for direct routing can be expressed as:

$$
E_{D R}=E_{t x}(s, h R)
$$

Substituting Eq. (10) into Eq. (23), results in:

$$
E_{D R}=s E_{a m p}\left(\alpha+(h R)^{2}\right)=s E_{a m p}\left(\alpha+h^{2} R^{2}\right)
$$

When comparing the two data transmission approaches, for the energy used in a multi-hop transmission approach to be smaller than that of a single-hop transmission approach, the condition of $E_{M H R}<E_{D R}$, has to be met, therefore: 


$$
s E_{a m p}\left(2 \alpha h+h R^{2}-\alpha\right)<s E_{a m p}\left(\alpha+h^{2} R^{2}\right)
$$

If the linear network is considered in terms of the strength of transmitter electronics, receiver electronics, and transmission amplifier, starting again with the multi-hop transmission approach. The expected energy consumption is:

$$
\begin{aligned}
E_{M H R} & =h E_{\text {tx }}(b, R)+(s-1) E_{r x}(h) \\
& =h\left(s E_{\text {elec }}+s R^{2} E_{\text {amp }}\right)+(s-1) s E_{r x} \\
& =s\left[\left(h E_{\text {elec }}+h R^{2} E_{\text {amp }}+s E_{\text {elec }}-E_{\text {elec }}\right)\right] \\
& =s\left[2 h E_{\text {elec }}-E_{\text {elec }}+h R^{2} E_{\text {amp }}\right] \\
& =s\left[h R^{2} E_{\text {amp }}+(2 h-1) E_{\text {elec }}\right]
\end{aligned}
$$

The direct transmission approach has the following:

$$
E_{t x}(s, R)=E_{t x}(s,(h R))
$$

Hence direct transmission energy consumption will become;

$$
E_{D R}=s E_{\text {elec }}+s(h R)^{2} E_{\text {amp }}
$$

Further simplified to;

$$
E_{D R}=s\left(E_{\text {elec }}+h^{2} R^{2} E_{\text {amp }}\right)
$$

Also comparing two data transmission approaches, for the energy used in a multi-hop transmission approach to be less than that of direct transmission approach, $E_{M H R}<E_{D R}$, therefore:

$$
\begin{gathered}
s\left[h R^{2} E_{\text {amp }}+(2 h-1) E_{\text {elec }}\right]<s\left(E_{\text {elec }}+\right. \\
\left.h^{2} R^{2} E_{\text {amp }}\right)
\end{gathered}
$$

Further simplified to:

$$
\frac{E_{\text {elec }}}{E_{\text {amp }}}>\frac{h R^{2}}{2}
$$

Where $\alpha$ is the amplification factor, such that;

$$
\alpha>\frac{h R^{2}}{2}
$$

\section{Computational and simulation experiments}

This work considered two different network scenarios for the computational and simulation experiments, these being the linear based WSHN and randomly distributed WSHN. In both network scenarios, this work adopted the parameters used in [33]. In the first part of the experiment, this work observed the variation of the average transmission cost of a grid-based WSHN for the distance between grid-based Wi-Fi nodes, the packet size of information and the number of Wi-Fi nodes in the enclosure (room). While in the second phase of the experiment, this work considers the comparison of the energy consumption of a direct and multi-hop communication in WSHM, where this work also observed the variation or behavior of different network types for number of nodes in the enclosure, packet size and the distance apart of the nodes' distribution in the enclosure. In both experiments, this work was limited to a maximum of 150 feet for the Wi-Fi modules transmission (indoor). However, the ESP 32 used can extend to 1kilometer $(3,280$ feet) [34], for which this work conducted experiments to see the viability in the said range of transmission. This is because this work is an application-specific to a building. The maximum current drawn is $260 \mathrm{~mA}$ at $2.4 \mathrm{GHz}$ operating frequency. This is in accordance with [33, 34].

\subsection{Average transmission cost of a grid-based WSHN}

This part of the paper shows the results of the average transmission cost for a typical WSHN deploying grid-based transmission in a grid network. As can be seen in Fig. 7, gotten using Eq. (13), it was observed that the cost of transmission measured in joules per feet $(\mathrm{J} / \mathrm{ft})$, is a function of packet size $(S)$ being sent, the distance between the smart devices, packet and the total number of hops the message makes to reach its destination. In Fig. 7(a), the impacts of transmission distance effects on the average cost of transmission is observed. For a small increase in transmission distance, the energy consumed per foot is obvious for each of the smart sockets or switches in the network. This justifies the relationship as in Eq. (13). In this, it is evidence that limiting the transmission distance in the network will favor the lifetime of the entire network due to the limited energy available per sensor node (smart device). For a typical Wi-Fi node (ESP 32) as in [34], each module can transmit at 150 feet indoor but up to $1 \mathrm{~km}$ outdoor without obstruction. For a high transmitting node, it comes with a high cost of energy consumption as can be seen in Fig. 7 (a). In Fig. 7 (b), is shown the relationship between average transmission cost and information packet size. This is like Fig. 7(a), as an increase in bits of information directly affects the energy consumed by the smart 
devices in the network. The effect of the packet size on the average transmission cost is also shown in Eq. (13), which is translated in Fig. 7 (b). In Fig. 7 (c), is shown the effect of the number of smart devices in the home network, which also correspond to the relationship in Eq. (13) as the average transmission cost is directly affected by the density of smart devices in the home network.

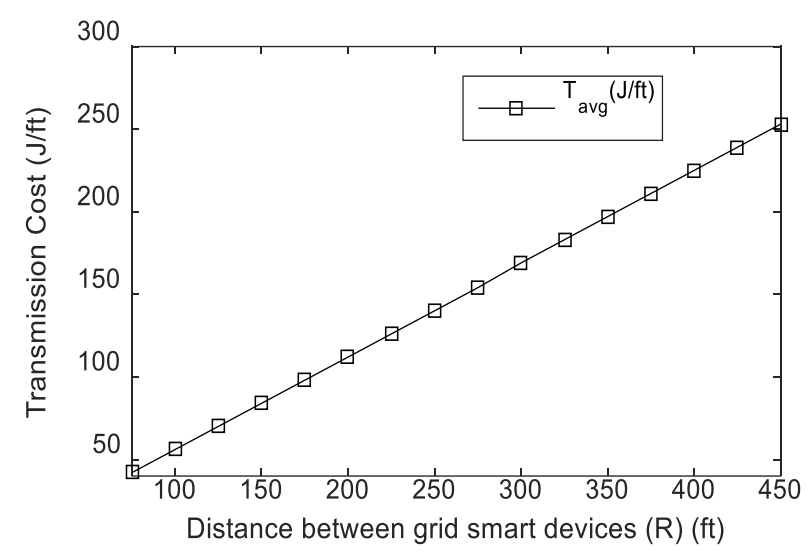

(a)

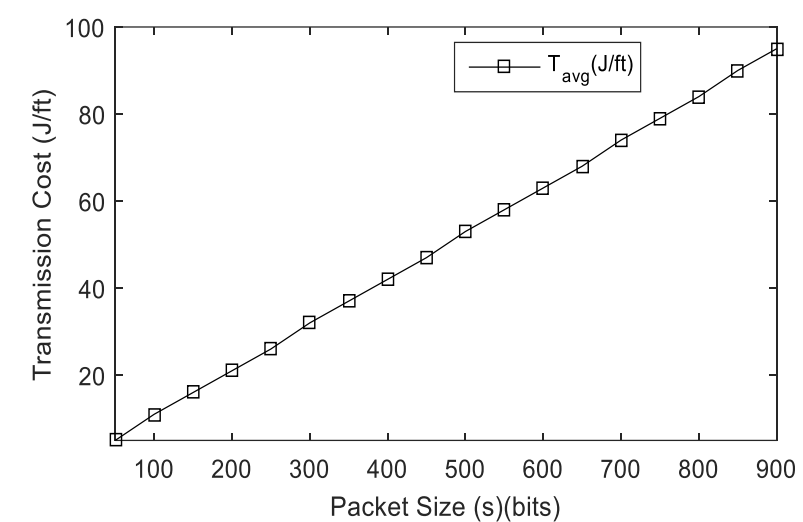

(b)

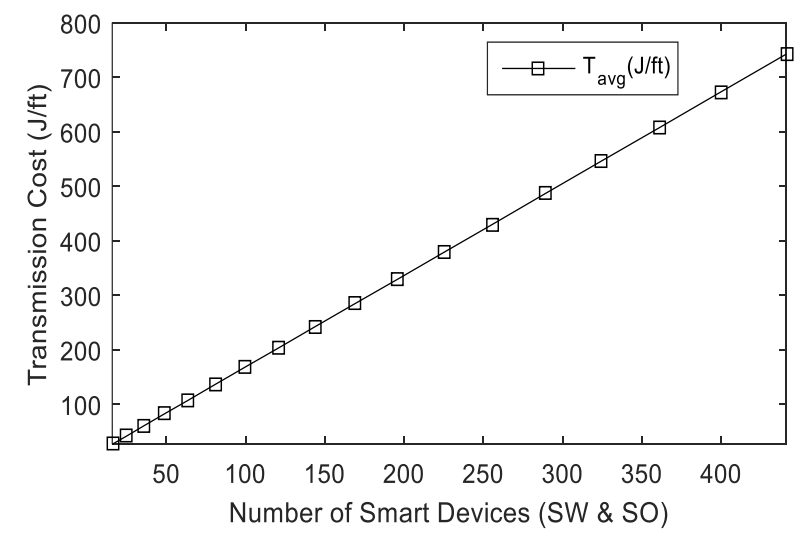

(c)

Figure. 7 Relationships of: (a) Transmission distance (R) (b) Packet Size (S), and (c) Number of Smart Devices (SW \& SO) on the Transmission cost for a grid-based wireless sensor home network

\subsection{Energy consumption of a direct and multi- hop communication in WSHN}

For the comparison of energy consumption for direct and multi-hop communication as given in Eqs. (23) and (24), for both network communication type, the results as shown in Fig. 8 (a)-(c), described the variation of consumed energy for the different network type: multi-hop and single-hop communication in WSHN to (a) packet size $(S)$, (b) transmission distance $(R)(\mathrm{ft})$, and $(\mathrm{c})$ network size (Hops). It was observed that there is a high network energy consumption when using single-hop communication compared to using multi-hop communication as the packet size is increased. This is shown in Fig. 8(a). In Eq. (23), it is evidence that the energy consumption of single-hop transmission is proportional to the square of the number of bits transmitted in the network during reception and transmission phase, which is an indicator of many bits transmitted per unit joule exhausted in the network. This refers to the fact that, for multi-hop communications, energy is saved due to the transmission of bits at a shorter distance. As can be seen in Fig. 8 (a), when the packet size value rises to 500 bits, the energy consumption per smart device for the single-hop communication approaches $50 \mathrm{~J}$ per second and rises to the value of $70 \mathrm{~J}$ per second at $700 \mathrm{bits}$, while it is at $12 \mathrm{~J}$ per second and $17 \mathrm{~J}$ per second for multi-hop communication. There is not much difference for the multi-hop (2J) as against 20J between 500bits and 700bits for direct communication. Furthermore, it is evident as can be seen in Fig. 8 (b), that, even with an increase in number of smart devices in the home network, the energy consumed using the multi-hop communication has no significant variation as it tends to remain almost the same value as the initial consumption for fewer smart devices in the home network, whereas, using the direct communication in the network, it is observed that, there is much consumption in the network as number of hops vary from 4 to 20 , the energy consumption is $79 \mathrm{~J}$ and 1986J respectively. But for multi-hop, it is $20 \mathrm{~J}$ and 99J respectively. There is a huge difference in the said number. This is to be expected to be that as the network size increases, there is an increase in transmission range, which does not favor single-hop home network communication. The multi-hop achieved this reduced energy conception as most of the smart devices are then engaged in helping other devices to route the information, hence, serving transmitters and routers in the network. In Fig. 8 (c), the impact transmission distance has on energy 


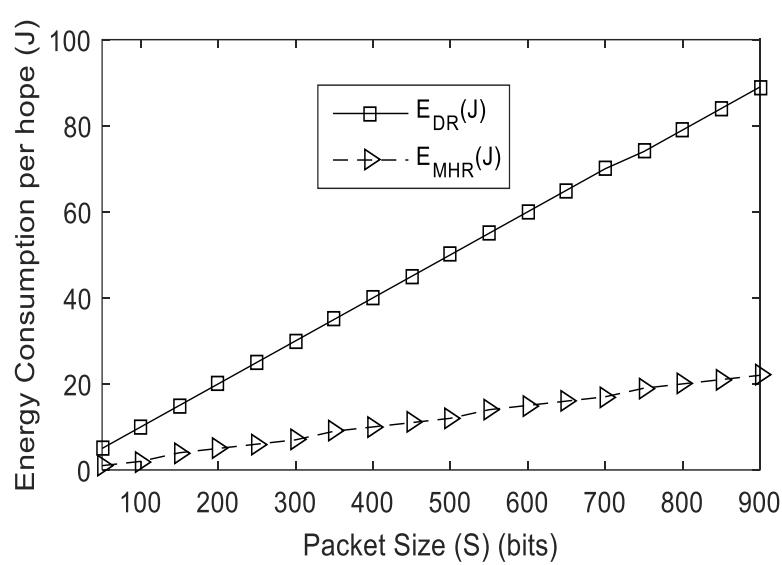

(a)

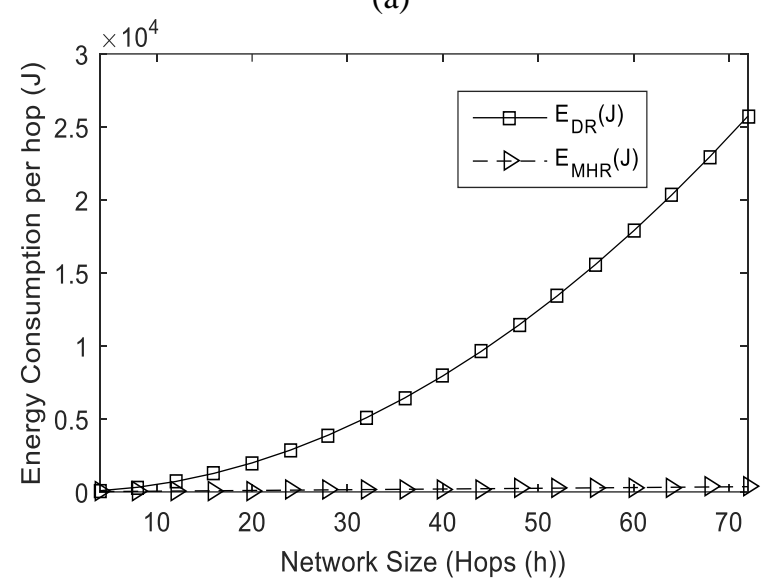

(b)

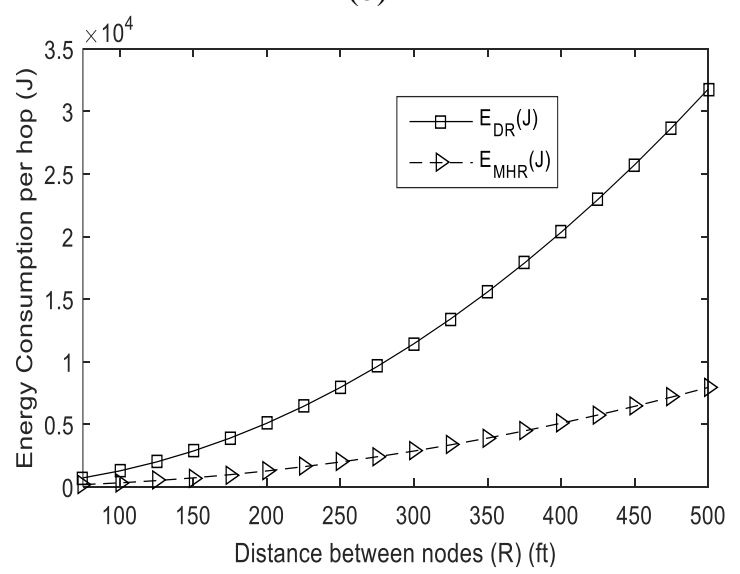

(c)

Figure. 8 Variation of Consumed Energy for the different network type: Multi-hop and Direct Communication in WSHN to: (a) Packet Size (S) (b) Network Size (Hops), and (c) Transmission distance (R) (ft)

consumption for the two-transmission type in a gridbased sensor home network is shown. This scenario does not differ from that of Fig. 8(a-b), as the variation of energy consumption, is also high for single-hop communication as compared to multihop for the variation of distance between the smart devices.

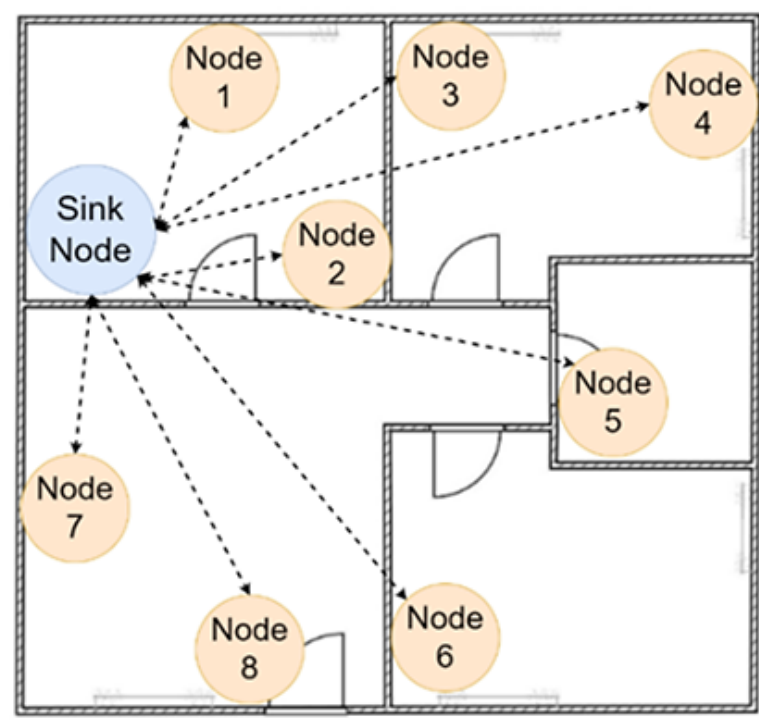

Figure. 9 Star topology (single hop) wireless sensor network

\section{WSHN implementation discussion}

Taking into consideration, the mathematical analysis and simulation results on wireless sensor networks carried out in sections three (3) and four (4). A single hop or star topology network is more suitable for the implementation of the proposed smart home system WSHN. Taking from the graphs of Fig. 8, it is evident that in most cases, using single-hop communication might cause excessive energy consumption in the network and that multihop communication is seen to be of preferable communication due to its lower energy consumption at a higher number of nodes, large packet size and longer distance between device.

However, it is also evident that at small distances, such as below 50ft, the differences in energy consumption between the two methods is less pronounced. Hence for applications of WSHN in houses and buildings where distances are typically small, using the single-hop topology and routing approach over multi-hop will not only have little comparable energy consumption but it will also reduce the complexity of the network and promote the responsiveness of the network since messages will travel directly to the sink node. Fig. 9 gives an example of the general structure and network topology to be implemented. All the network sensor nodes, these being the smart devices, will connect and communicate directly with the sink node, which is the smart hub.

For such a mostly event-driven application, the advantage of reduced delay time in direct communication will provide users with a satisfactory response of the system since there will be a little pause from the time a user initiates an 
event and when the system responds to the event or stimuli.

\section{Conclusion}

This paper, analytically modeled a home-based wireless sensor network for application in smart switches and sockets, serving as sensor nodes, and smart hub as a sink node. Each of the nodes (smart switches/sockets and or hub) is embedded with Wi$\mathrm{Fi}$ enable communication technology. However, the current practice in comparing energy consumption in the WSHN systems is by measuring delay, throughput and energy consumption performance through experience and real-time measurement approach. This work carefully covered models of node distribution and connectivity, energy and network reliability model and the computation and simulation of the home network. The analytical method used in the WSHN topology in a line-based and grid-based sensor home network is on energy consumption for varying network parameters, with a description of each of the network. The results of our analytical model show a variation of network energy consumption for single-hop and multi-hop communication for reference purposes in future WSHNs planning. It was evident that, in most cases, using single-hop communication might cause excessive energy consumption in the network. It becomes more pronounced when the distance apart of the smart devices is above 50feet or the network nodes is above 20; more also, when the packet size is above 50 bits. In this case, multi-hop communication is seen to be of preferable communication at a higher number of nodes, large packet size and longer distance between devices. However, if the network size is small, there is little difference in the energy consumption between the two methods and it is in such networks where single-hop is preferred due to the reduced complexity of the network structure and small delay time of which promotes responsiveness of the network. In future, we intend to simulate WSHN using emerging wireless technologies such as BLE, LoRa and Wi-Fi 6 to contrast and compare their energy consumption in WSHNs.

\section{Acknowledgments}

The authors wish to acknowledge the financial support from the Botswana International University of Science and Technology (BIUST) throughout the research.

\section{References}

[1] M. Zungeru, L.-M. Ang, and K. P. Seng, "A formal mathematical framework for modeling and simulation of wireless sensor network environments utilizing the hill-building behavior of termites," Simulation, Vol. 89, No. 5, pp. 589-615, 2012.

[2] L.K. Ketshabetswe, A.M. Zungeru, M. Mangwala, J.M. Chuma, and B. Sigweni, "Communication Protocols for Wireless Sensor Networks: A Survey and Comparison”, Heliyon, Elsevier, Heliyon, Article number e01591, 2019.

[3] L.K. Ketshabetswe, A.M. Zungeru, J.M. Chuma, and M. Mangwala, "Swarm Intelligence-Based Communication Protocols for Wireless Sensor Networks", Critical Developments and Applications of Swarm Intelligence. Yuhui Shi (Eds.): Chapter 11, pp. 271-300, 2018.

[4] K. Sha and W. Shi, "Modeling the Lifetime of Wireless Sensor Networks", Sens. Lett., Vol. 3, pp. 126-135, 2005.

[5] T. Zhao and Q. Zhao, "Lifetime Maximization Based on Coverage and Connectivity in Wireless Sensor Networks", Signal Process. Syst., Vol. 57, pp. 385-400, 2009.

[6] K. Biswas, V. Muthukkumarasamy, X.-W. Wu, and K. Singh, "An Analytical Model for Lifetime Estimation of Wireless Sensor Networks", IEEE Commun. Lett., Vol. 19, No. 9, pp. 1584-1587, 2015.

[7] Wang, L. Xing, V. M. Vokkarane, and Y. Sun, "Reliability analysis of wireless sensor networks using different network topology characteristics", In: Proc. of 2012 International Conference on Quality, Reliability, Risk, Maintenance, and Safety Engineering, 2012.

[8] W. Dargie, "A Quantitative Measure of Reliability for Wireless Sensor Networks", IEEE Sens. Lett., vol. 3, No. 8, pp. 1-4, 2019.

[9] A.K. Singh, S. Rajoriya, S. Nikhil, and T. K. Jain, "Design constraint in single-hop and multi-hop wireless sensor network using different network model architecture", In: Proc. of Communication Automation International Conference on Computing, pp. 436-441, 2015.

[10] C.-M. Yu, M.-L. Ku, and C.-W. Chang, "Hybrid multi-hop/single-hop opportunistic transmission of WSNs", In: Proc. of 2017 IEEE International Conference on Consumer Electronics - Taiwan (ICCE-TW) pp. 111-112, 2017.

[11] V. Sharma and D. S. Saini, "Performance Investigation of Advanced Multi-Hop and 
Single-Hop Energy Efficient LEACH Protocol with Heterogeneous Nodes in Wireless Sensor Networks", In: Proc. of 2015 Second International Conference on Advances in Computing and Communication Engineering, pp. 192-197, 2015.

[12] C. Peng, C. Chen-Nee, and L. Xin, "Energyaware node placement in wireless sensor networks", In: Proc. of IEEE Global Telecommunications Conference, Vol. 5, pp. 3210-3214, 2004.

[13] Y. Li, S. Yin, L. Liu, S. Wei, and D. Wang, "Battery-Aware MAC Analytical Modeling for Extending Lifetime of Low Duty-Cycled Wireless Sensor Network", In: Proc. of 2013 IEEE Eighth International Conference on Networking, Architecture and Storage, pp. 297-301, 2013.

[14] L. M. Rodrigues, C. Montez, G. Budke, F. Vasques, and P. Portugal, "Estimating the lifetime of wireless sensor network nodes through the use of embedded analytical battery models", J. Sens. Actuator Netw., Vol. 6, No. 2, 2017.

[15] L. M. Rodrigues, C. Montez, R. Moraes, P. Portugal, and F. Vasques, "A temperaturedependent battery model for wireless sensor networks", Sensors, Vol. 17, No. 2, p. 422, 2017.

[16] S. Galmés, "Optimal routing for time-driven EH-WSN under regular energy sources", Sensors, Vol. 18, No. 11, p. 4072, 2018.

[17] H. Sharma, A. Haque, and Z. A. Jaffery, "Maximization of wireless sensor network lifetime using solar energy harvesting for smart agriculture monitoring", Ad Hoc Netw., Vol. 94, p. 101966, 2019.

[18] S. Galmés and S. Escolar, "Analytical model for the duty cycle in solar-based EH-WSN for environmental monitoring", Sensors, Vol. 18, No. 8, p. 2499, 2018.

[19] Y. Zhang, Y. Wei, M. Song, and J. Song, "RAODV: Rate Aware Routing Protocol for WiFi Mesh Networks", In: Proc. of IET International Conference on Wireless Mobile and Multimedia Networks, Vol. 2006, pp. 44-44, 2006.

[20] H. Farman, H. Javed, B. Jan, J. Ahmad, S. Ali, F.N. Khalil, and M. Khan, "Analytical network process based optimum cluster head selection in wireless sensor network", PLOS ONE, Vol. 12, No. 7, pp. 1-28, 2017.

[21] Y. Zhang, M. Fromherz, and L. Kuhn, "Routing modeling application simulation environment (RMASE)",
https://www2.parc.com/isl/groups/era/nest/Rma se/ (2011, accessed 21 January 2018).

[22] M. Meghji and D. Habibi, "Transmission power control in multi-hop wireless sonsor network", In: Proc. of 2011 Third International Conference on Ubiquitous and Future Networks, pp.25-30, 2011.

[23] R. Moreno, A. Bermúdez, R. Casado, and A. Robles-Gómez, "SensGrid: Modeling and simulation for wireless sensor grids", Simulation, Vol. 88, No. 8, pp. 972-987, 2012.

[24] W. R. Heinzelman, A. Chandrakasan, and H. Balakrishnan, "Energy-efficient communication protocol for wireless microsensor networks", In: Proc. of the 33rd Annu. Hawaii Int. Conf. Syst. Sci., Vol. 1, No. c, p. 10, 2000.

[25] M. Panda and M.R. Patra, "Modeling radio channels for CSMA-MAC based wireless sensor networks", In: Proc. of International Journal of Computer Applications, Vol. 9, No. 6, pp. 6-11, 2010.

[26] M. Meghji, D. Habibi, "Transmission power control in multihop wireless sensor network", In: Proc. of the 2011 Third International Conference on Ubiquitous and Future Networks, pp. 25-30, 2011.

[27] W.R. Heinzelman, A. Chandrakasan, and H. Balakrishnan, "Energy-efficient communication protocol for wireless microsensor network", In: Proc. of the 33rd Annual Hawaii International Conference on Systems Science, Vol. 2, pp. 3005-3014, 2000.

[28] R. Moreno, R.-G Antonio, B. Aurelio, and C. Rafael, "SensGrid: modeling and simulation for wireless sensor grids", Simulation: Transactions of the Society for Modelling and Simulation International, pp. 1-16, 2012.

[29] L.-H. Hsu and C.-H. Lin, "Graph Theory and Interconnection Networks", CRC Press, Taylor and Francis Group, 2009.

[30] Z.A Khan, C. Belleudy, and M. Auguin, "Analytical Model for Energy Consumption Analysis in Grid Based Wireless Sensor Networks", In: Proc. of the International Conference on New Technologies, Mobility and Security, pp. 1-5, 2009.

[31] S. Toumpis and A.J. Goldsmith, "Capacity regions for wireless ad hoc networks", IEEE Transaction on Wireless Communication, Vol. 2, pp. 736-748, 2003.

[32] A.M. Zungeru, L.-M. Ang, S.R.S. Prabaharan, and K.P. Seng, "Radio Frequency Energy Harvesting and Management for Wireless Sensor Networks", Energy Scavenging and Optimization Techniques for Mobile Device, $V$. 
Hrishikesh, and G-M. Mountean (Eds.) USA: CRC Press, Taylor and Francis Group, Chapter 13, pp. 341-367, 2011.

[33] A.l. Diego, "Analysis and evaluation of viable features for an IEEE 802.11n/ac self-optimizing solution", Master of Science Thesis, KTH Royal Institute of Technology, Stockholm, Sweden, 2017.

[34] A. Alasdair, "Long-Range WiFi for the ESP32", Hackster.io, 2018. 